\title{
Grin and bear it! Neural consequences of a voluntary decision to act or inhibit action
}

\author{
Elisa Filevich · Patrick Haggard
}

Received: 20 October 2011/Accepted: 5 September 2012/Published online: 25 September 2012

(C) The Author(s) 2012. This article is published with open access at Springerlink.com

\begin{abstract}
Action inhibition is an important part of everyday human behaviour. Most previous studies of action inhibition have focussed on stop-signals. Here, we consider the case where participants themselves decide to inhibit, or not inhibit, a prepotent action. Participants received electric stimulation that elicited an itchy feeling on the wrist. If they made a hand withdrawal movement, this would interrupt the stimulation, and halt the itch. In a factorial design, participants were given external instructions to withdraw their hand when they felt the itch, or to inhibit the natural withdrawal response, and bear the itch. In another condition, they were asked to internally choose between withdrawal and inhibition of withdrawal. Eventrelated potentials revealed differences between processing of the sensory consequences of internally decided and externally-instructed action and inhibition decisions. Specifically, potentials evoked by itchy stimuli were enhanced in internally decided inhibition trials, as compared to externally instructed inhibition trials. In contrast, processing of itchy stimuli was reduced in internally decided action trials, as compared to externally instructed action trials. These results show that internal decisions lead to different perceptual processing of the consequences of action and inhibition and suggest that features of decision processes can be measured via their consequences.
\end{abstract}

Keywords Internal inhibition - Itch - Action inhibition · Self-control

E. Filevich $(\bowtie) \cdot$ P. Haggard

Institute of Cognitive Neuroscience,

University College London, London, UK

e-mail: e.filevich@ucl.ac.uk

\section{Introduction}

Inhibition of action is an important part of human behaviour. Most people recognize the familiar situation of having to appeal to their self-control to overcome urges to eat, drink, buy or choose something. A common reason for this internal inhibition is our knowledge that we would regret the action in the future, however, attractive it may be in the present.

\section{Externally instructed inhibition}

Most research on the capacity to inhibit actions has studied the neural processes triggered by an exogenous instruction to inhibit (Logan 1994; Verbruggen and Logan 2008). In these tasks, participants generally make simple prepotent actions, such as manually responding to GO signals. They occasionally and unpredictably are presented with an additional NO GO signal or STOP signal, which instructs them to inhibit the prepotent response, and withhold the instructed action. Several decades of research using these paradigms have confirmed the importance of the frontal lobes in inhibitory processing (Aron et al. 2004).

However, there is an emerging consensus on the need to study inhibition with richer and more complex tasks (Aron 2011). Brass and Haggard (2007) coined the term 'intentional inhibition' to refer to inhibition that arises internally, from an internal decision to withhold an action, rather than from an external signal (Brass and Haggard 2007). Here, we use the more neutral term 'internal inhibition'. This concept partly overlaps with the concept of willpower developed in behavioural social psychology (Baumeister et al. 2007), in that both have the effect of preventing or delaying inappropriate actions. 
Inhibitory self-control

Recent experimental work has given rise to the idea that willpower, or self-control, is a general capacity, or limited resource analogous to the body's physical energy (Hagger et al. 2010). The inhibitory mechanisms associated with willpower relate to a general state in which inhibition is continuously present, until exhausted. However, internal inhibition might also involve a temporally specific decisional process, analogous to external inhibition triggered by a stop signal. For example, it has been argued that people may withhold an action at the last possible moment (Libet 1985). Therefore, in an event-related framework for internal inhibition, inhibition should appear not only as a general, sustained mental process, but also as a specific, clearly timed event. That is, the decision to inhibit may be taken in the context of a specific stimulus, and may have specific consequences. Treating inhibition as event-related would allow the neural mechanisms to be measured more precisely.

Experimental constraints raise significant methodological problems, notably for ecological validity. Most previous experimental studies of 'free will' have not given participants clear reasons for choosing to act or inhibit and thus have low ecological validity (Libet et al. 1983; Brass and Haggard 2007). Perhaps, the need for methodological simplicity has meant that decisions, urges, values and consequences of actions have been conspicuously missing in these paradigms.

\section{Measuring the consequences of inhibition}

One recent experiment suggests that the sensory consequences of action could be useful to describe the processes of action that were in fact inhibited. Shocks delivered to participants' fingers were perceived as weaker after action inhibition triggered by an external stop signal, as compared to a passive detection task (Walsh and Haggard 2010), suggesting that some characteristics of voluntary action are maintained even if the action itself is inhibited. An experimental framework to study internal inhibition of action requires three components. First, there must be a reason to perform an action. Second, the participant must make an internal decision to inhibit that action on some occasions. Third, there must be some way of measuring the internal processes associated with inhibition, rather than merely recording whether an action occurred or was inhibited.

Resisting the urge to scratch an itch

Most people can recognize the urge to scratch an itch. Not scratching can be extremely effortful, and it can make the itchy feeling more intense. We developed an experimentally controlled version of this situation to create a paradigm that would meet these requirements.

In this ecologically valid task, reasons for actions were provided by delivering on each trial itchy and unpleasant stimuli that could be avoided by doing a hand movement. In this way, decisions to act or inhibit would have meaningful consequences. Internal decisions about action and inhibition were allowed in some trials, whereas clear external instructions were provided in other trials. Electroencephalography (EEG) was recorded to measure brain activity.

Specifically, participants were either instructed or had to decide whether to move their arm to avoid an unpleasant itchy sensation, or to inhibit the urge to move the arm, and withstand the itch. Thus, a strong motivation to act was introduced.

We compared event-related potentials (ERP) for situations in which inhibition of action followed either from an external instruction or from an internal decision. The ERP method allowed us to focus specifically on the sensory processing of the itchy stimuli themselves. Sensory processing of itchy stimuli produces a strong event-related potential (Mochizuki et al. 2008). We took advantage of the good temporal consistency of these known potentials to apply EEG techniques to compare these different processes across conditions.

Our main hypotheses were as follows: First, a decision to execute or inhibit action will influence subsequent sensory processing. Second, and most importantly, this influence will vary with the source of the decision: internal decisions would have different 'downstream' effects on sensory processes from externally instructed decisions.

\section{Materials and methods}

\section{Participants}

Sixteen naïve paid healthy volunteers ( 9 females, mean age $25.3 \pm 5$ years) participated in the experiment. Participants with sensitive skin were excluded from taking part in the study. Procedures were approved by the UCL Research Ethics Committee and were in accordance with the principles of the Declaration of Helsinki. One participant was excluded after participation due to excessive blinking.

Participants sat comfortably at a table and $60 \mathrm{~cm}$ away from a computer screen. Both hands rested comfortably on the table, so that their right index finger would rest on a force-sensitive resistor (FSR) (Active Robots Ltd, Somerset, UK), connected to a computer. 


\section{Electrical stimuli}

Itchy stimuli were delivered using previously established methods (Mochizuki et al. 2008) with some adaptations. Briefly, each electrode consisted of four pairs of stainless steel wire $0.1 \mathrm{~mm}$ in diameter. Each pair formed a cross and was placed approximately $2 \mathrm{~mm}$ away from the next pair. Current for each electrode was supplied by a Digitimer medical stimulator (Digitimer Ltd, Hertfordshire, England) and flowed through all wires. The reference electrodes (cathodes) were placed $1 \mathrm{~cm}$ laterally to each itch electrode. Current was delivered through the electrodes in square pulses of 2-ms duration at $50 \mathrm{~Hz}$. A stepwise procedure was used to determine the current intensity necessary to elicit an unpleasantly itchy sensation in each participant. The current was explicitly set to a level that would rather be avoided, but would still be bearable. Participants were asked to rest their right hand on a forcesensitive pad. Electrical stimulation would flow through the electrodes on their left forearm only as long as the pad was touched by the right hand. As soon as the participant withdrew their hand from the pad, stimulation would stop and the itchy sensation would cease. Thus, participants could stop an unpleasant sensation on their left wrist by actively withdrawing their right hand from a resting position. Critically, whilst participants held their hand in place on the pad they actively inhibited the urge to act.

As expected from previous studies (Mochizuki et al. 2009), participants reported a strong habituation of the itchy sensation with repeated stimulation. Therefore, two separate itch electrodes were placed on the left wrist. Stimulation lasted for $3 \mathrm{~s}$ altogether and consisted of three 1-s-long shocks, alternating from one electrode to the other. The first electrode to be stimulated (proximal or distal to the wrist) alternated across trials.

Task and experimental design

The experiment consisted of 10 blocks of 40 trials each, and lasted 80-90 min. Each trial was organized as follows: (see Fig. 1) a black fixation cross appeared over a grey background for a variable duration of between 2 and $3 \mathrm{~s}$. Two visual stimuli (called V1 and V2) were presented sequentially. These signalled respectively the start of each trial, and the instructions for a given trial. V1 was a green circle subtending $1.5^{\circ}$ at a distance of approximately $60 \mathrm{~cm}$, appearing for $250 \mathrm{~ms}$ to mark the initiation of the trial. Participants were asked to prepare a right-hand movement as soon as V1 appeared. The fixation cross then appeared again on screen for $2 \mathrm{~s}$, until a second circle appeared (V2), again for $250 \mathrm{~ms}$. The luminosity of V1 and V2 was balanced with an heterochromatic flicker test with an independent set of 5 participants. This was done to adjust the intensity of the visual stimuli independently of their absolute luminance, but depending on participants' sensitivity (Wyszecki and Stiles 1982). V2 was of the same size as V1 and could be of three different colours. If V2 was green, participants should remove their right hand as soon as they felt the shock on their left, thus terminating the shock.

The green V2 represented an 'external go' condition, in which participants were instructed by an external instruction to perform a movement. If V2 was red, participants were asked to endure the shock and were not to move their hands. Hence, the red V2 represented the 'external no go' condition, in which participants were instructed again by an external instruction, to avoid doing any movement despite having the desire to do so.

Alternatively, V2 could be yellow, in which case the participants were asked to decide whether they would endure the shock or withdraw their hand as soon as they felt the shock, thus avoiding prolongation of the itchy

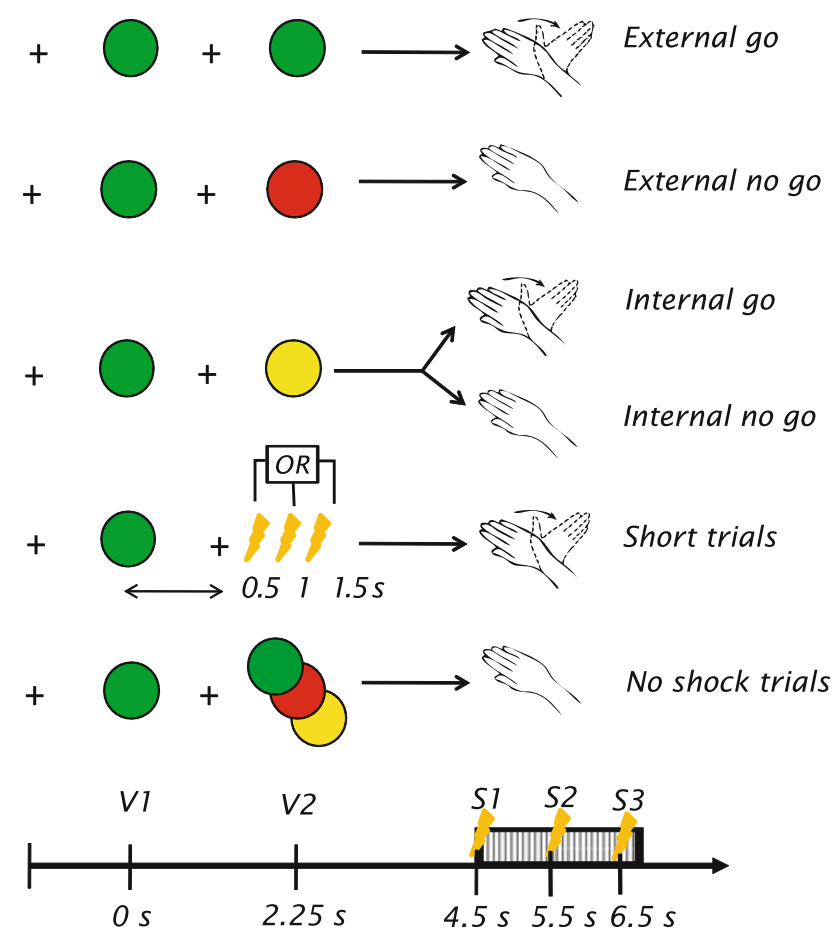

Fig. 1 Experimental task. A visual warning sign (V1) was presented for $250 \mathrm{~ms}$. After a 2-s interval, an instruction sign (V2) was presented, also for $250 \mathrm{~ms}$. After a second 2-s interval, three consecutive electrical shocks were delivered at $50 \mathrm{~Hz}$ and for a maximum of $3 \mathrm{~s}$, generating an itchy feeling on the participants' wrist. If participants moved their hand, they would interrupt the itchy feeling. Green- and red-coloured V2 corresponded to external instructions to move the hand or not. Yellow V2 allowed the participants themselves to choose between the two possible action outcomes. Short trials in which V2 was replaced at variable times by a surprise shock were presented to encourage and probe motor preparation. Catch trials with no shock delivered were presented to ensure that participants were waiting for the shock to execute their decision 
sensation. In the yellow V2 condition, participants had to decide whether each trial would be a ' $g o$ ' or ' $n o$ go'. Hence, depending on whether the participant decided to move or not, the yellow V2 could produce an 'internal go' or 'internal no go' condition. Participants were encouraged to choose to withdraw their hand in roughly $50 \%$ of the yellow V2 trials. After each block of 40 trials, they received feedback if the rate of withdrawal was higher than $70 \%$ or lower than $30 \%$ of the trials. Because participants preferred to withdraw their hands whenever they had the choice, this manipulation ensured that the overall number of trials were comparable across conditions for the EEG analysis. The block-by-block feedback and relatively loose boundaries around $50 \%$ were included to prevent participants from developing a very strict strategy.

In this way, the experiment followed a factorial $2 \times 2$ experimental design, with the factors of source (internal/ external) and outcome (go/no go). Critically, there was no behavioural difference between the internal and external conditions, so any differences found in the recorded neural signal associated with the internal or external sources of decision would necessarily reflect differences in the processing of internal vs. external decisions.

Two additional conditions were included for methodological reasons: $15 \%$ 'catch' trials (without a shock) were presented to ensure that participants waited for the first shock before executing their internal decision or instruction and did not simply predict its onset. All three V2 colours were followed by catch trials with equal probability. In addition, to encourage movement preparation, $25 \%$ of 'short' trials were included. In these trials, itch stimulation was delivered at either $0.5,1.0$ or $1.5 \mathrm{~s}$ after V1, in contrast with the normal time of 2,250 ms. Participants were asked to withdraw their hand from the FSR as quickly as possible in these cases.

The mean intensity at which participants reported to feel an unpleasant but bearable itchy sensation was $0.36 \pm$ $0.14 \mathrm{~mA}$ at the beginning of the experiment for both electrodes. After each block, intensity was readjusted if the stimulation was perceived as too painful or too mild. Intensity never exceeded $0.4 \mathrm{~mA}$, and by the end of the experiment, the mean intensity at which subjects perceived the itchy sensation was $0.38 \pm 0.14 \mathrm{~mA}$ and $0.38 \pm 0.15 \mathrm{~mA}$, respectively, for each one of the stimulators.

Electrophysiological recordings and signal analysis

A SynAmps amplifier system and Scan 4.3 software (Neuroscan, El Paso, TX) were used to record EEG data. Activity from fourteen scalp electrodes was recorded (F3, Fz, F4, FC3, FCz, FC4, C3, Cz, C4, P3, Pz, P4, O1, O2, according to the 10-20 system). The reference electrode was $\mathrm{AFz}$, and the ground electrode was placed on the chin. All electrode impedances were kept below $5 \mathrm{~K} \Omega$. The left and right mastoids were recorded. Horizontal electrooculogram (EOG) was recorded from bipolar electrodes placed on the outer canthi of each eye, and vertical EOG was recorded from bipolar electrodes placed above and below the right eye. EEG signals were amplified and digitized at $500 \mathrm{~Hz}$.

EEG data were analysed with EEGLAB software (Delorme and Makeig 2004). Data were first re-referenced to the linked mastoids. Because long epochs $(8.25 \mathrm{~s})$ were defined, data were digitally high-pass filtered over $0.5 \mathrm{~Hz}$ to remove low frequency drifts. In addition, we computed the amplitude of event-related potentials (ERPs) as peak amplitude values. A $30 \mathrm{~Hz}$ low-pass filter was applied to the data (Mochizuki et al. 2008). Continuous EEG data were time-locked to the trial start (stimulus V1) and baselined to the period of $250 \mathrm{~ms}$ to $150 \mathrm{~ms}$ prior to the onset of V1. To avoid artefacts due to eye blinks, trials were discarded if the bipolar recording of EOG exceeded $\pm 80 \mu \mathrm{V}$ at any point during the epoch. The mean percentage of rejected trials was $22 \%$. This value is relatively high, but perhaps unsurprising given the long epochs and the unpleasantness of the experience. The components in the evoked response were identified by inspection of the grand-average pooling across all conditions. For each component identified in the grand average, the time of maximum amplitude of the individual average was determined and the values for each participant in that time point were computed.

\section{Results}

Behavioural results

Participants rarely moved their hands in catch trials (mean \pm SD commission errors $0.70 \pm 0.91 \%$ ).

On average, within the internal trials, participants decided to withdraw their hands (internal go) on $46 \pm 5 \%$ (mean $\pm \mathrm{SD}$ ) of the trials. To measure the extent of preparation of the action to withdraw the hand, we calculated the average RT to withdraw the hand after receiving an itchy shock. The RT was compared across the internal and external go conditions (for which the withdrawal movement could be anticipated and prepared) and the average of all 'short' trials, in which the shocks occurred without a prior V2 warning signal, therefore not allowing for movement preparation (Fig. 2).

A repeated measures ANOVA revealed a main effect of condition $\left(F_{1,15}=19.38, p<0.001\right)$. RTs were longer for short trials as compared to both internal $g o$ and external $g o$ conditions. Paired comparisons revealed significant differences between the internal go condition and the short trials $\left(t_{15}=-6.22, p<0.001\right)$ and between the external 


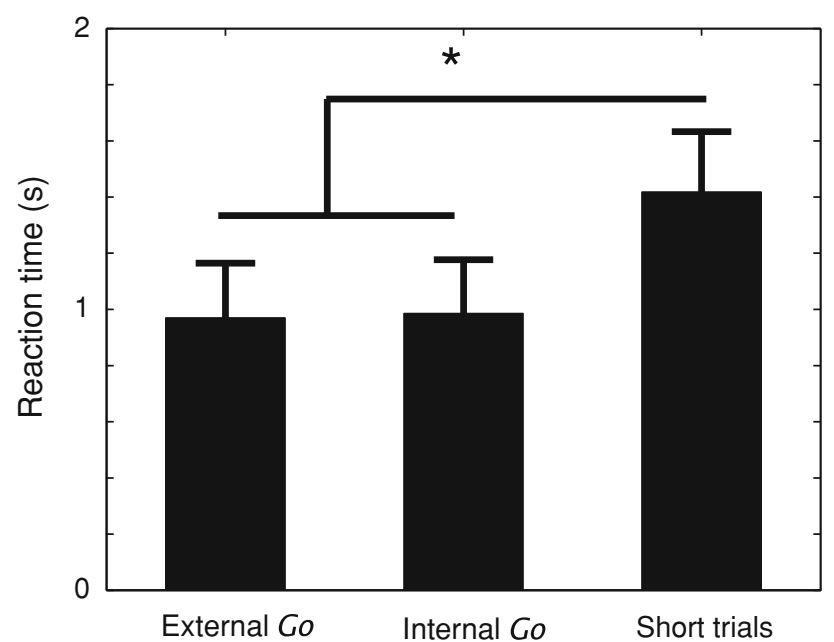

Fig. 2 Behavioural results. Hand withdrawal times for the movement conditions. No difference is observed between the two movement conditions, in which the time of shock was highly predictable. Longer withdrawal times are observed in 'short' trials, suggesting that motor preparation had occurred in both internal and external go trials

go condition and the short trials $\left(t_{15}=-5.25, p<0.001\right)$. No significant differences were found between the RTs for the internal and external go conditions $\left(t_{15}=-0.54\right.$, $p=0.59$ ). These results suggest that there was movement preparation in the two go conditions that was less efficient in the short trials. It is also possible that go trials had shorter RTs due to the timing of the shocks becoming more predictable. Our design cannot distinguish between these two possibilities.

We observed relatively long RTs (of around $1 \mathrm{~s}$ ). Such long reaction times may partly reflect the peculiar nature of this stimulation. An interesting feature of this itch stimulus is the lack of a discrete perceptual onset at the start of the shock-train. Short trains do not produce any sensation at all. At the intensities we used, sensory perception begins only some time after the onset of the stimulation. Because the reaction time is measured from the onset of the stimulus, the reaction time is artificially increased by delay, which we attribute to accumulation of signals in perceptual areas.

These behavioural results did not change when we excluded the participant that was excluded from the ERP analysis due to excessive blinking.

\section{ERP results}

After blink rejection, an average of $42 \pm 16$ trials (SD) were recorded for the internal go condition, and $41 \pm 17$ trials were recorded for the internal no go condition. $42 \pm 13$ trials were recorded for the external go condition, and $49 \pm 13$ trials fell into the external no go

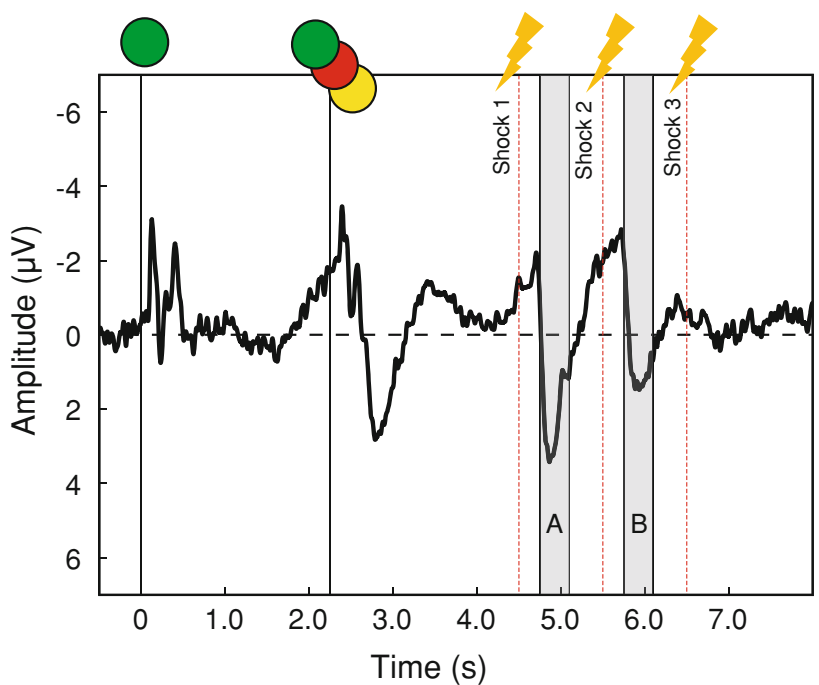

Fig. 3 EEG results Grand average across all the four main experimental conditions (internal and external go and no go) for electrodes $\mathrm{C} 3, \mathrm{Cz}$ and $\mathrm{C} 4$. Long ( $8 \mathrm{~s}$ ) epochs were visually inspected to identify two main periods of interest: response to shock $1(A)$ and response to shock $2(B)$. The response to shock 3 was greatly attenuated and was therefore not analysed. EEG trace is plotted negative-up

condition. The grand-average ERPs were displayed timelocked to V1, to reveal the sequence of sensorimotor events in each epoch. Figure 3 shows the grand-average trace at $\mathrm{C} 3, \mathrm{Cz}$ and $\mathrm{C} 4$ pooled across all conditions. There is a stereotyped response to the onset of both V1 and V2. Importantly, although V1 and V2 are physically similar, only V2 carries information about the subsequent task instructions. Accordingly, the neural processing of V1 differs strongly from that of V2, with only V2 eliciting a strong positivity peaking at around $580 \mathrm{~ms}$ after V2 onset. There is a characteristic negativity preceding V2, recalling the CNV (Lumsden et al. 1986). This negativity starts roughly $800 \mathrm{~ms}$ before the onset of the first shock is visible in the grand average across conditions. Finally, the neural response to the three consecutive shocks is apparent. A marked positive-going component occurs in response to each of the three shocks, peaking at around $400 \mathrm{~ms}$ after shock onset.

The key ERP components evoked by the first two shocks are indicated by shaded areas $A$ and $B$ in Fig. 3. Figure 3 shows an average of all conditions, including both $g o$ and no go conditions. Therefore, whilst the first shock was always delivered, and acted as a GO signal, the second and third shocks were not experienced if participants withdrew their hand. In addition, the third shock was hardly perceived, even on no go trials, due to habituation. It was therefore not included in the analysis. Peak amplitudes for each of these events were analysed for each condition separately. 
Our analysis focussed on the effects of source of decision (internal or external) and decision outcome ( $g o$ or no go) on the neural activity evoked by the shocks.

Our analyses were restricted to central and parietal electrodes, because we were interested in assessing the neural consequences of inhibition over sensory processing, rather than the frontal mechanisms that cause inhibition itself (Aron et al. 2004).

\section{Pre-shock components}

We first examined the response to the instruction cue. We chose a time window of $350-550 \mathrm{~ms}$ after V2. In accordance to the topographical distribution in the half-point of the chosen time window, electrodes $\mathrm{C} 3, \mathrm{Cz}, \mathrm{C} 4, \mathrm{P} 3, \mathrm{Pz}$ and $\mathrm{P} 4$ were averaged. A $2 \times 2$ ANOVA of the peak amplitudes revealed a trend for a main effect of outcome $\left(F_{1,14}=3.42, p=0.08\right)$, with a stronger $\mathrm{V} 2$ positivity in the no go conditions. There was no main effect of source $\left(F_{1,14}=0.39, p=0.53\right)$ or interaction effect $\left(F_{1,14}=\right.$ $0.03, p=0.59)$.

We then examined the preparatory activity the V2 instruction and before shock 1. An inspection of the grand average (Fig. 3) shows that there is an RP/CNV component before shock 1(Kornhuber and Deecke 1965; Walter et al. 1964). Topographical maps showed that this component was maximal between $\mathrm{C} 3$ and $\mathrm{Cz}$. It was measured as the mean amplitude during the $200 \mathrm{~ms}$ prior to the shock for the average of these two electrodes. A $2 \times 2$ ANOVA of the RP/ CNV amplitude revealed a main effect of outcome $\left(F_{1,14}=5.00, p=0.042\right)$. This arose because preparatory negativity was significantly stronger for go trials compared with no go trials. However, there was no main effect of source $\left(F_{1,14}=2.86, p=0.11\right)$, and no significant interaction between source and outcome $\left(F_{1,14}=0.57, p=0.46\right)$.

A Average response to shocks

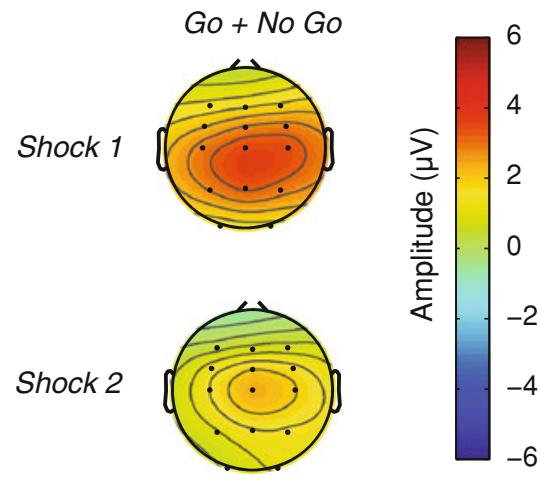

Fig. 4 Topographical distribution of the neural responses to the shocks. The topographical maps for the response to shock 1 were calculated at 4,900 ms, and the response to shock 2 were calculated at $5,900 \mathrm{~ms}$ from V1 onset. a Average of all conditions. The topography
Evoked responses to the shocks

We examined the average topography of the response to shocks 1 and 2 (see Fig. 4a). As expected from previous results, the average topographical maps show that the response to the both shocks is focused on the central electrodes. Based on this topography, peak amplitudes for the analysis were obtained from the average of electrodes $\mathrm{C} 3, \mathrm{Cz}$ and $\mathrm{C} 4$.

We also examined the differences between the external and internal conditions for both go and no go trials separately (see Fig. $4 \mathrm{~b}$ ). The topographical maps show large differences in the left hemisphere, ipsilateral to the shocks but contralateral to the movement. This suggests that this difference is related to motor preparation. The most parsimonious, albeit speculative, interpretation is that go trials show a stronger (more negative) RP-like movement preparation component for internal than for external trials. This leads to a positivity observed in the electrodes contralateral to movement in the external-internal subtraction.

\section{Evoked response to shock 1}

The topography and peak amplitude of the response to shock 1 is shown in Fig. 5 (highlighted section $A$ in Fig. 3).

A $2 \times 2$ ANOVA of the average revealed no main effect of outcome $\left(F_{1,14}=0.06, p=0.80\right)$ nor main effect of source $\left(F_{1,14}=0.01, p=0.92\right)$ but a significant interaction effect $\left(F_{1,14}=19.433 p=0.001\right)$, showing a crossover form in ure 5C. Post hoc $t$ tests revealed that the neural response evoked by shock 1 was greater in external $g o$ than in internal go trials $\left(t_{14}=3.39, p=0.004\right)$. Conversely, the response evoked by shock 1 in external no go trials was weaker than that evoked by the internal no go trials $\left(t_{14}=-2.22, p=0.04\right)$.

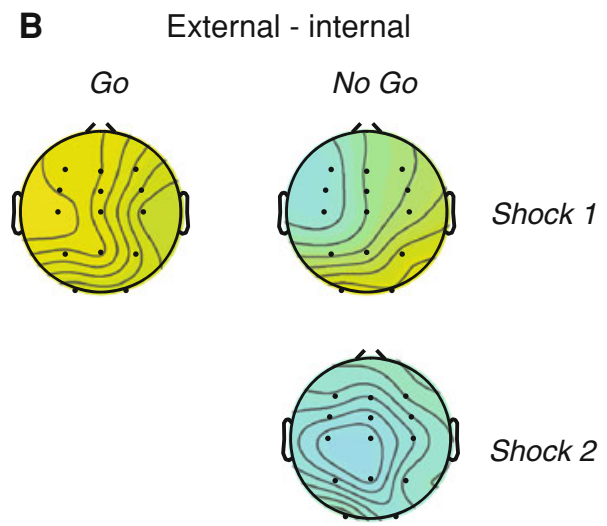

includes an average of four conditions (external go, external no go, internal go, internal no go). Shock 2 includes external no go and internal no go conditions only. b Differences between external and internal conditions, for both go and no go trials 
A
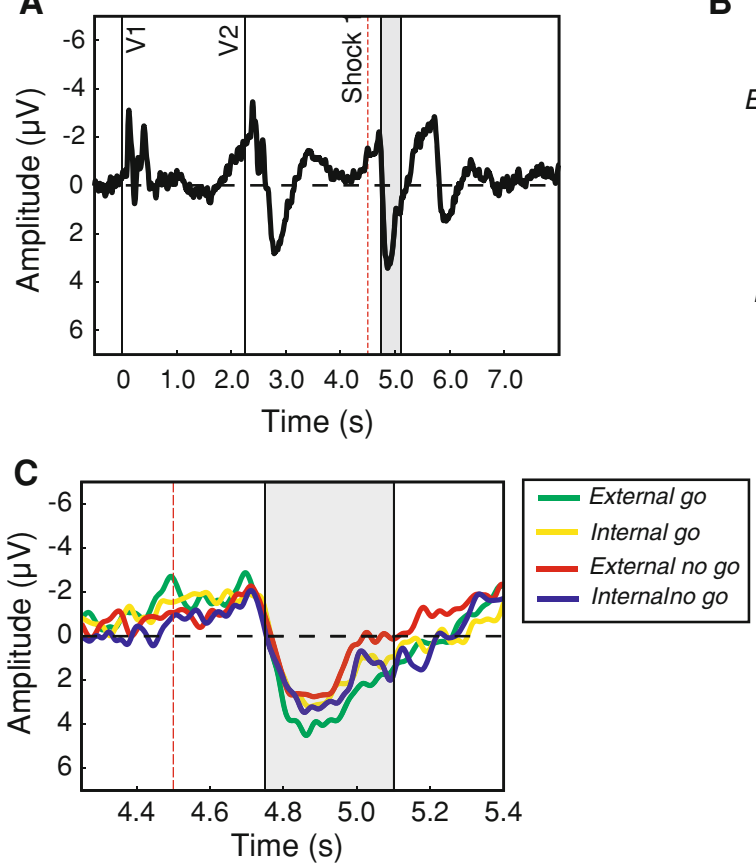

B

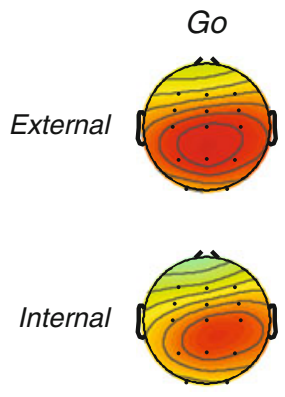

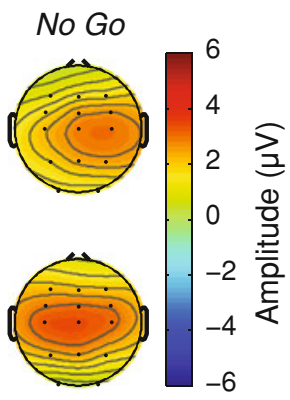

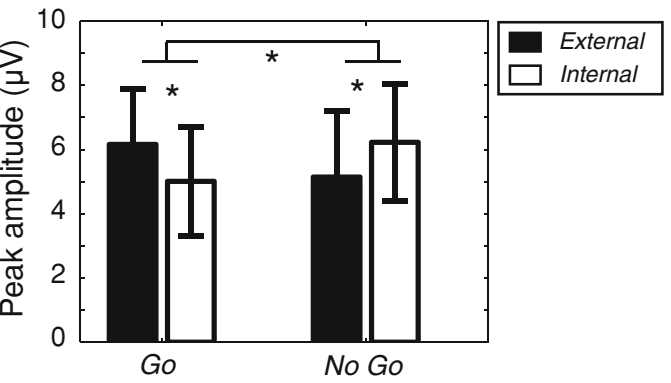

Fig. 5 Neural response to shock 1 (a) Time window $(4,750-5,100$ $\mathrm{ms}$ ) in which the amplitude of the response to shock 1 was measured. The vertical dashed line indicates the time of shock onset. b Scalp distribution at 4,900 ms. c Detail of time window of interest, showing the averaged trace for electrodes $\mathrm{C} 3, \mathrm{Cz}$ and $\mathrm{C} 4$ for each condition. d Mean of maximum amplitude for each subject within the selected

\section{Evoked response to shock 2}

Because the mean RT to withdraw the left hand was generally shorter than $1 \mathrm{~s}$, shock 2 was generally not delivered in trials where participants made the withdrawal action with their right hand. Hence, the analysis of the ERP to shock 2 was confined to no go trials, in which participants did not withdraw their hand, but resisted the full train of shocks. The topography and peak amplitudes for the no go conditions are shown in Fig. 6 and match that of shock 1 (see also highlighted section $B$ in Fig. 3). A paired $t$ test comparison revealed a significant difference between the two no go conditions, with the internal no go again showing a stronger potential in response to itchy shock compared to external no go $\left(t_{14}=-2.33, p=0.03\right)$.

To compare the neural response to shocks 1 and 2, a $2 \times 2$ ANOVA of the no go responses with the factors shock and source of decision was carried out. Results showed no main effect of shock $\left(F_{1,14}=1.202, p=0.29\right)$, a main effect of decision source $\left(F_{1,14}=7.114, p=0.01\right)$, with internal no go trials showing stronger ERP than external no go trials. There was no interaction effect $\left(F_{1,14}=0.21, p=0.65\right)$.

Shock 3 was not analysed because participants reported at debriefing that they rarely felt it. This reflected a strong time window, for the average of electrodes $\mathrm{C} 3, \mathrm{Cz}$ and $\mathrm{C} 4$. Error bars show confidence intervals. A significant interaction effect emerges $(p<0.01)$. Post hoc $t$ tests show a crossover effect. Shock 1 ERPs are stronger for external go trials as compared to internal go trials $(p<0.05)$, whereas ERPs to external no go trials are weaker than those for internal no go trials $(p<0.05)$

habituation, and the evoked potentials were correspondingly weak.

\section{Discussion}

A paradigm that generates strong urges to make hand withdrawal movements was used to study the inhibitory functions involved in self-control over one's own actions. Further, situations of internally and externally triggered inhibition were compared within the same context. Participants were either instructed, or decided for themselves, whether to withdraw their right hand from a response pad, thus terminating a train of unpleasantly itchy shocks delivered via a customized electrode to the left forearm.

This paradigm therefore allowed us to study the consequences of inhibition, following both internal decisions and external signals. Specifically, we investigated how internal inhibition influenced neural processing of subsequent itchy shocks, in comparison to externally instructed inhibition. In both these conditions, there is no overt behaviour. However, the source of the decision to inhibit differs between these conditions. We show that the source of inhibitory decisions has an important structuring effect on subsequent sensory experience. This is, to our knowledge, the first 
A

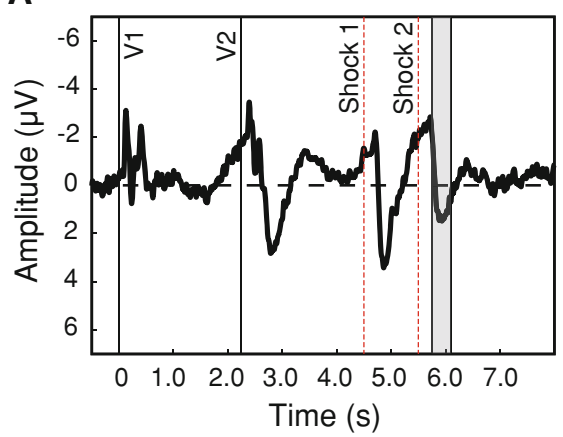

C

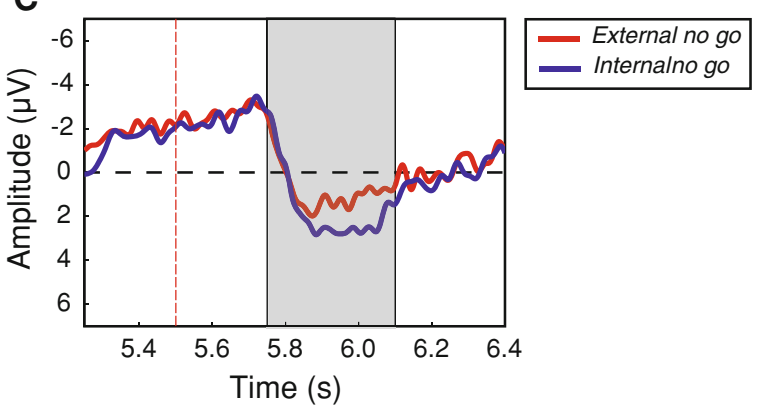

B
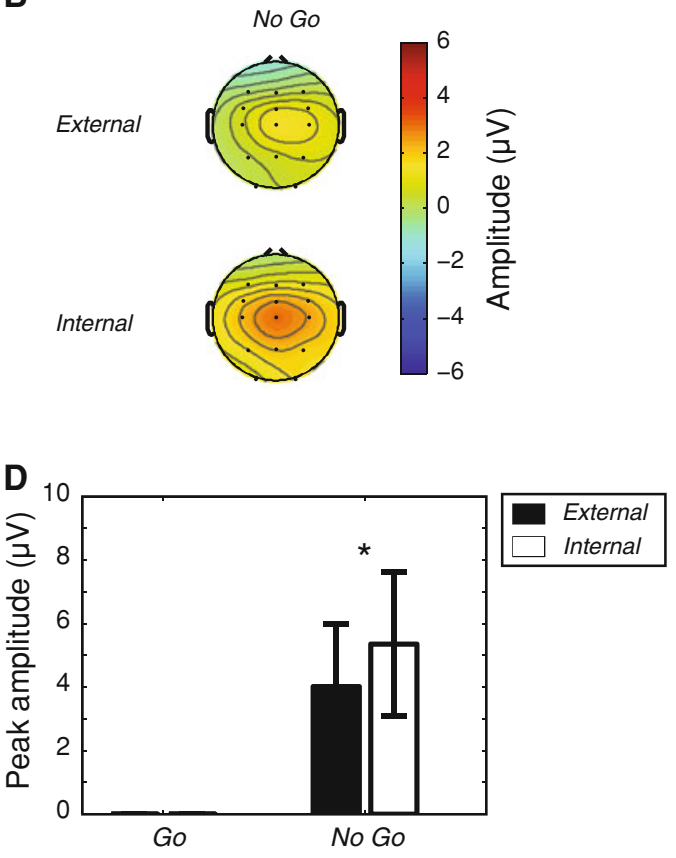

Fig. 6 Neural response to shock 2. Because shock 2 was only fully delivered in inhibition trials, only no go trials are analysed. a Time window $(5,750-6,100 \mathrm{~ms})$ used to measure the amplitude of the response to shock 2. The vertical dashed line indicates the time of shock onset. b Scalp distribution at $5,900 \mathrm{~ms}$. c Detail of time window of interest, showing the averaged trace for electrodes $\mathrm{C} 3, \mathrm{Cz}$

study to address the consequences of self-control and internal inhibition of action on somatosensory processing.

\section{Ecological validity}

Our experiment used aversive stimulation to induce a strong urge to act. Unlike previous studies of internal inhibition, we therefore made the choice between action and inhibition motivationally significant. We combined this with the conventional comparison between externally instructed and internally decided actions (Lau et al. 2004), in which participants are asked to 'freely choose' between two alternatives roughly half the time. The motivational element of the experiment may seem to conflict with the free selection element: if itchy shocks were truly aversive, participants should choose to avoid them. Our participants' willingness to accept the experimental instructions perhaps lead them to trade shocks for money (Talmi et al. 2009). However, the balance of positive and negative affect indubitably plays a major role in shaping everyday action choices (Damasio and Dolan 1999). Notwithstanding these general motivations, our participants made a fresh choice on every internally decided trial, whether to act or inhibit on that trial. The random intermingling of internally decided and externally instructed trials was designed to and $\mathrm{C} 4$ for each condition. $\mathbf{d}$ Mean of maximum amplitude for each subject within the selected time window, for the average of electrodes $\mathrm{C} 3, \mathrm{Cz}$ and $\mathrm{C} 4$. Error bars show confidence intervals. As in shock 1, external no go trials evoke a weaker response to shock 2 than an internal no go trials $(p<0.05)$

discourage them from preparing sequences of 'free' choices extending over several trials. For these reasons, our study may have an ecological validity lacking in previous studies of internal inhibition.

Neural responses to the electrical shocks

A marked positive-going component occurs in response to each of the three shocks, peaking at around $400 \mathrm{~ms}$ after shock onset. Previous studies using similar stimuli (Mochizuki et al. 2009) had reported slower positive components, peaking at around $900 \mathrm{~ms}$. We can only speculate on the sources of this difference. Mochizuki et al. associated the long latency of the evoked response with C-fibre activation, because of their slow conduction speed. Indeed, itch sensation has been mainly associated with C-fibres (Handwerker 2010). However, some A $\delta$-fibres have also been associated with itch in monkeys (Schepers et al. 2008) and humans (Ringkamp et al. 2011). Thus, we speculate that our pulses may also have stimulated both $\mathrm{C}$-fibres and the faster-conducting $\mathrm{A} \delta$-fibres. Activation of $\mathrm{A} \delta$-fibre can lead to both sharp and burning pain. The joint activation of different fibre populations may have produced the distinct sharp itchy feeling that our participants experienced. 
In our paradigm, participants always received the first shock and received the second and third shocks only if they chose to inhibit the action of withdrawing their arm. The third shock was perceived very weakly and accordingly produced only a small ERP. Previous reports (Mochizuki et al. 2009) suggested that diminishing neural activity evoked by itch reflected habituation effects. Decrease in saliency for repeated stimuli has been widely reported (Legrain et al. 2011).

Go and no go trials cannot easily be compared directly, because several different factors may contribute to the differences between their neural correlates. Crucially, go trials involve motor preparation, whilst no go trials do not. We therefore do not draw any strong conclusions about differences between go and no go trials. However, the comparing internal and external sources of action decisions is possible, within both the go and no go condition, because the motor activity is balanced between the two sources of decision.

The neural response for shock 1 produced lower ERP amplitudes when participants internally decided to withdraw their hands, as opposed to when they were externally instructed to do so. The neural response to shock 2 cannot be evaluated in the case of movement conditions, because when the hand has been withdrawn, no further shocks are delivered. However, in the case of no go trials, the neural trace of an aversive stimulus that could have been avoided, but was not, could be informative about the mechanisms of inhibition. In these trials, the response to the first and second shock can be evaluated. The neural response to both the first and second shocks was significantly larger when participants underwent the shocks as a result of their own internal decision, rather than as a result of an external instruction.

Thus, whilst action trials showed smaller ERPs to itchy stimuli in the internally decided go trials than in externally instructed go trials, inhibition trials showed the opposite effect. We found stronger ERPs for internally decided no go trials as compared with externally instructed no go trials. We speculate that this interaction effect reflects differences in allocation of attention strategies between go and no go trials. In our paradigm, participants presumably attended to the shocks when they needed to react quickly (i.e. in go conditions). In no go trials, the shock is an aversive but inevitable stimulus that is therefore not attended. In no go conditions, participants may have preferred to 'think of something else' and try to completely ignore the shocks. Interestingly, in internally decided trials, this effect is reversed. That is, the attention allocation towards the aversive stimuli in go trials and away from aversive stimuli in no go trials would have been less efficient in the internal conditions, according to this view. It has been suggested (Fleming et al. 2009) that internal decisions for actions are less definitive, and easier to change, than externally instructed actions. Could this explain the differences we found between the processing of aversive stimuli in internal and external no go trials? If Fleming et al. are correct that internal decisions to act or inhibit still leave open the counterfactual possibility, we speculate that consequences of internal decisions might be strongly processed because of feelings of regret for the missed opportunity of doing otherwise.

In other words, external trials have clear instruction, and there is a clear correct answer. This is not the case with internal trials, in which any course of action would be correct. Therefore, in line with results reported by Fleming et al. (2009), attention allocation in internal trials may represent an 'intermediate' situation between the two extremes: allocation of attention towards an aversive stimulus in external go trials and allocation of attention away from an aversive stimulus in external no go trials.

An alternative, but closely related interpretation, relates to motor processing. In this study, we aimed at investigating action inhibition indirectly by addressing the sensory processing of its consequences. However, motor and sensory processes were not temporally segregated in our task. Moreover, EEG techniques do not allow us to unequivocally identify the sources of the modulation of the shock components as either clearly sensory or clearly motor. Thus, it remains possible that our results reflect movement-related processing. Because we did not investigate the periods before action directly, or EEG components that are classically related to action, the hypothesis that our peak ERP amplitude is affected by motor processes remains speculative. The influence of motor preparation on the shock component amplitude is unclear. Importantly, however, this interpretation remains compatible with the 'intermediate' account of internal decisions that we suggest above.

Intermediate ERP peak amplitudes may therefore reflect intermediate levels of motor preparation. In particular, whereas external go trials are associated with a high levels of motor preparation, internal go trials seem to present lower levels of motor preparation, closer to the no go conditions. In turn, external no go trials are presumably associated with lower levels of motor preparation because no action should occur in no go trials. Internal no go trials present higher levels of action preparation, closer to go trials.

Crucially, in line with the results reported by Fleming et al. (2009), we suggest that internal decisions for action represent situations that are less committed to than instructed decisions, and therefore may be easier to change than corresponding external decisions. If the attention allocation account is correct, our results may interestingly extend this interpretation from the purely motor processing 
addressed by Fleming et al. to the sensory processing of decision consequences.

Inhibition of action can take two rather different forms depending on its time-course. First, it can be a rather tonic behavioural control, related to the concept of willpower (Baumeister et al. 2007; Vohs and Schmeichel 2003). For example, someone who exerts self-control over their eating behaviour may need to continuously inhibit the urge to eat. These inhibitory processes are continuous and ongoing, rather than discrete and precisely timed. Inhibitory selfcontrol may also appear in a more phasic form, as a lastminute inhibition of specific and discrete action impulses (i.e. veto) (Libet 1999). The type of inhibition required in our task lies somewhere on the continuum between these two extremes. Because some trials required inhibition and others required a quick action, the task was designed to encourage phasic, discrete inhibition, rather than generalized, tonic self-control. The present results therefore address the concept of self-control or 'willpower' in a novel experimental way.

Here, by focusing on the consequences of internal inhibition on subsequent sensory processing, we have provided an event-related measure of internal inhibition, suitable for comparison with instructed inhibition.

\section{Conclusion}

Everyone recognizes the agonizing situation of wanting to respond to an aversive stimulus, but forcing oneself not to. For example, we know that we should not scratch an itch mosquito bite, or drop a cup of coffee that feels too hot. In such cases, we internally inhibit a prepotent action. Our results support the concept of a specific process of internal inhibition, by showing the strong structuring effect of voluntarily withholding action on subsequent sensory processing. When participants internally inhibited an action which would have spared them from itchy shocks, the neural response to those shocks was stronger than when the same choice was made externally. The consequence of a decision to inhibit seems to be more strongly processed than the consequence of an instructed inhibition. When we are given an external instruction, no other action choice is available, and it is relatively easy to grin and bear an aversive stimulus. In contrast, when we ourselves decide what to do, the sensory consequences of our decisions are experienced all the more strongly. This may be because we could have avoided these consequences by choosing otherwise.

Acknowledgments We thank Hideki Mochizuki and Ryusuke Kakigi for help with supplying itch electrodes, and Simone Kuhn for advice and assistance. This work was supported by the Wellcome
Trust, an Overseas Research Students award from the British Council $[\mathrm{EF}]$, a European Science Foundation-European Collaborative Research Project/Economic and Social Science Research Council grant (RES-062-23-2183), and by a Leverhulme Trust Major Research Fellowship to PH.

Open Access This article is distributed under the terms of the Creative Commons Attribution License which permits any use, distribution, and reproduction in any medium, provided the original author(s) and the source are credited.

\section{References}

Aron AR (2011) From reactive to proactive and selective control: developing a richer model for stopping inappropriate responses. Biol Psychiatr 69(12):e55-e68

Aron AR, Robbins TW, Poldrack RA (2004) Inhibition and the right inferior frontal cortex. Trends Cognit Sci 8:170-177

Baumeister RF, Vohs KD, Tice DM (2007) The strength model of self-control. Curr Dir Psychol Sci 16:351-355

Brass M, Haggard P (2007) To do or not to do: the neural signature of self-control. J Neurosci 27:9141-9145

Damasio A, Dolan RJ (1999) The feeling of what happens. Nature 401:847-848

Delorme A, Makeig S (2004) EEGLAB: an open source toolbox for analysis of single-trial EEG dynamics including independent component analysis. J Neurosci Methods 134:9-21

Fleming SM, Mars RB, Gladwin TE, Haggard P (2009) When the brain changes its mind: flexibility of action selection in instructed and free choices. Cereb Cortex 19:2352-2360

Hagger MS, Wood C, Stiff C, Chatzisarantis NLD (2010) Ego depletion and the strength model of self-control: a meta-analysis. Psychol Bull 136:495-525

Handwerker HO (2010) Microneurography of pruritus. Neurosci Lett 470(3): 193-196

Kornhuber HH, Deecke L (1965) Hirnpotentialänderungen bei Willkürbewegungen und passiven Bewegungen des Menschen: Bereitschaftspotential und reafferente Potentiale. Pflüg Arch European J Physiol 284(1):1-17

Lau HC, Rogers RD, Ramnani N, Passingham RE (2004) Willed action and attention to the selection of action. NeuroImage 21:1407-1415

Legrain V, Iannetti GD, Plaghki L, Mouraux A (2011) The pain matrix reloaded: a salience detection system for the body. Prog Neurobiol 93:111-124

Libet B (1985) Unconscious cerebral initiative and the role of conscious will in voluntary action. Behav Brain Sci 8:529-539

Libet B (1999) Do we have free will. J Conscious Stud 6:47-57

Libet B, Gleason CA, Wright EW, Pearl DK (1983) Time of conscious intention to act in relation to onset of cerebral activity (readiness-potential). The unconscious initiation of a freely voluntary act. Brain. J Neurol 106(Pt 3):623-642

Logan GD (1994) On the ability to inhibit thought and action: a users' guide to the stop signal paradigm. In: Dagenbach D, Carr TH (eds) Inhibitory processes in attention, memory and language. Academic Press, San Diego, CA, pp 189-239

Lumsden J, Howard RC, Fenton GW (1986) The contingent negative variation $(\mathrm{CNV})$ to fear-related stimuli in acquisition and extinction. Int J Psychophysiol 3:253-261

Mochizuki H, Inui K, Yamashiro K, Ootsuru N, Kakigi R (2008) Itching-related somatosensory evoked potentials. Pain 138:598603 
Mochizuki H, Inui K, Tanabe HC et al (2009) Time course of activity in itch-related brain regions: a combined MEG-fMRI study. J Neurophysiol 102:2657-2666

Ringkamp M, Schepers RJ, Shimada SG, Johanek LM, Hartke TV, Borzan J, Shim B et al (2011) A role for nociceptive, myelinated nerve fibers in itch sensation. J Neurosci 31(42):14841-14849

Schepers RJ, Johanek LM, Hartke TV, Shim B, Borzan J, Meyer RA, Ringkamp M (2008) A subpopulation of A-delta nociceptors in monkey is vigorously activated by cowhage spicules. Soc Neurosci Abstr 34:170.3

Talmi D, Dayan P, Kiebel SJ, Frith CD, Dolan RJ (2009) How humans integrate the prospects of pain and reward during choice. J Neurosci 29:14617-14626

Verbruggen F, Logan GD (2008) Response inhibition in the stopsignal paradigm. Trends Cognit Sci 12:418-424
Vohs KD, Schmeichel BJ (2003) Self-regulation and the extended now: controlling the self alters the subjective experience of time. J Pers Social Psychol 85:217-230

Walsh E, Haggard P (2010) Somatosensory effects of action inhibition: a study with the stop-signal paradigm. Exp Brain Res 204:465-473

Walter WG, Cooper R, Aldrige VJ, McCallum WC, Winter AL (1964) Contingent negative variation: an electric sign of sensorimotor association and expectancy in the human brain. Nature 203:380-384

Wyszecki G, Stiles WS (1982) Color science: concepts and methods, quantitative data and formulae. Wiley, New York 